\title{
Pensée tragique et pensée providentielle du mal
}

\section{Bernard Sichère}

\section{(2) OpenEdition \\ Journals}

\section{Édition électronique}

URL : http://journals.openedition.org/shakespeare/1579

DOI : 10.4000/shakespeare.1579

ISSN : 2271-6424

Éditeur

Société Française Shakespeare

Édition imprimée

Date de publication : 1 novembre 1997

Pagination : 163-179

Référence électronique

Bernard Sichère, "Pensée tragique et pensée providentielle du mal », Actes des congrès de la Société française Shakespeare [En ligne], 15 | 1997, mis en ligne le 01 janvier 2007, consulté le 21 avril 2019. URL : http://journals.openedition.org/shakespeare/1579; DOI : 10.4000/shakespeare.1579 


\section{PEN S É E TR A G I Q U E E T P E N S É E PRO V ID E N T IE L L E D U M A L}

Tout en vous remerciant de m'accueillir parmi vous, je me dois de faire état d'emblée de ma perplexité et de mes craintes. Je suis philosophe, ce qui n'est pas nécessairement bien vu de tous, et je ne suis pas spécialiste de Shakespeare et de l'Angleterre élisabéthaine comme mon ami Richard Marienstras, à qui je dois ma présence ici pour le meilleur et pour le pire. S'il a tenu à ma présence, c'est probablement, comme j'ai eu le pressentiment depuis un temps déjà au fil de nos conversations, que le regard du philosophe que je suis sur cet univers qui est le sien ne lui est pas indifférent. Je vais donc parler d'abord en philosophe de ce qui me paraît la différence (sinon la contradiction) entre une pensée tragique du mal (en l'occurrence la pensée des Grecs) et une pensée providentialiste (en l'occurrence, la pensée ou la théologie chrétienne). Mais ce n'est pas seulement en philosophe que je m'exprimerai malgré tout : si j'aime Shakespeare, ce n'est pas parce que je trouverais en lui de quoi alimenter par ailleurs une philosophie, c'est parce qu'il est un des plus grands poètes d'Occident, et spécifiquement un poète dramatique. Pour le dire plus nettement, ces questions de philosophe à propos du mal m'intéressent ici dans la mesure où elles vont à la rencontre de ce qui, dans le théâtre de Shakespeare, me donne à penser. Qu'il y ait bel et bien une pensée de Shakespeare, voilà ce dont je n'ai jamais douté, et je n'ai jamais douté non plus que cette pensée fût inséparable de la forme dramatique et poétique qui est celle du théâtre shakespearien. C'est précisément la question que je désire formuler et traiter devant vous, une question dont je m'étonne d'ailleurs qu'elles ne me soit pas venue plus tôt : s'il est vrai qu'il y a une pensée du mal dans Shakespeare et que cette pensée ne procède pas, comme la 
philosophie, par concepts, mais est intrinsèquement liée à la forme théâtrale, à la forme de ce théâtre-poème dont on peut dire que Shakespeare est l'inventeur, comment évaluer cette pensée en regard de ce qui jadis, chez les Grecs, s'est formulé comme pensée du mal interne à la forme spécifique de la tragédie ? Car il ne fait pas de doute que le drame shakespearien est l'héritier en connaissance de cause de cette dernière. La question est alors de savoir ce qu'il en est de cet héritage, ce qui en lui marque une continuité (Shakespeare penserait le mal en tragique au sens grec du terme) ou éventuellement une discontinuité voire une hétérogénéité (comment peut-on admettre que Shakespeare pense le mal en tragique, dans la suite de la pensée tragique des Grecs, s'il est vrai qu'il appartient par ailleurs à un monde chrétien, ordonné de part en part à partir du dogme et du kérygme chrétiens ?).

Vous me pardonnerez de revenir tout d'abord sur quelques aspects essentiels de la pensée tragique du mal telle que les Grecs de l'époque classique (disons les contemporains de Sophocle) ont pu l'élaborer. Une première donnée semble certaine, que nous devons concéder sans hésiter au philosophe Heidegger : la pensée grecque, contrairement à une solide tradition, n'est pas une pensée qui part de l'homme (s'il est vrai en revanche quelle «se tourne vers» lui), ce n'est pas une pensée que nous puissions ranger de près ou de loin sous la rubrique de ce que nous appelons depuis notre Renaissance «l'humanisme», ce n'est pas une pensée qui pense à partir de l'homme, mais une pensée qui pense à partir du primat de l'Être. Ce primat est donné dans le mot kosmos : il y a antériorité absolue d'un ordre fixe et hiérarchisé de l'univers, qui «était là» depuis toujours et au sein duquel est assignée à l'homme sa place ou sa part (moira). Ce primat est donné également dans le mot «dieux» (oi theoi) au pluriel : au commencement et avant les hommes il y a les dieux, les Lumineux, Ceux qui sont toujours (athanatoi), et c'est à partir d'eux seulement, en regard d'eux, qu'il est possible de poser les mortels, nous autres hommes qui, étant soumis au temps, à la corruption, à la mort, ne sommes pas vraiment ce que nous sommes. Or la question du mal se pose là d'emblée, dans cette antériorité des Immortels et dans ce primat assuré du kosmos. D'une part il y a, dans la disposition même de ce Kosmos, une part d'ombre : il y a depuis toujours guerre à l'intérieur de l'Être. D'autre part, cette guerre s'actualise dans le rapport des dieux et des hommes : s'il est vrai que les dieux sont ceux qui principalement tournent vers nous leur face de lumière dans la guise d'une bénédiction (kharis), ils peuvent aussi, pour des raisons qui nous échappent, se détourner de nous et inverser leur puissance de bénédiction en pouvoir de malédiction. Cette malédiction s'avère, pour le sujet qu'elle frappe, sous l'emblème du deinon, de la terreur et du terrible, ou de l'atè, de la ruine et du désastre. La dimension du mal surgit donc comme une énigme et non seulement comme un fait, comme l'énigme qu'un homme peut être pour lui-même dès lors qu'il se trouve arraché à ce qui semblait constituer 
son assise ou sa place au sein de la commune humanité, et soumis au risque extrême de la perte de soi dans cette région du «terrible» qui défie la pensée, les mots, la limite. Le sujet tragique est ainsi celui qui, dans l'affrontement à la puissance opaque de la ruine, s'abolit lui-même. Trois caractères de cette puissance maléfique sont à retenir : tout d'abord, elle est voulue obscurément par «les dieux» (par n'importe quels dieux, de grands dieux dont la puissance bénéfique peut s'inverser en maléfice: Apollon, Dionysos), et de telle manière que leur décret ne pourra pas ne pas s'accomplir; ensuite, cette puissance de ruine n'est pas pensable dans les termes de la libre décision personnelle (le mot destin, moira, l'exclut) ; enfin elle n'est pas à penser non plus dans le registre de l'existence individuelle mais dans celui d'un mal qui concerne, par delà l'individu, une famille, un clan, une «maison» (oikos).

Mais cela ne suffit pas encore à penser la dimension du mal mise en jeu par la tragédie : ce serait oublier, en effet, et le cadre réel à l'intérieur duquella représentation tragique est possible, et l'horizon de pensée qui constitue son interrogation. Ce cadre, nous le savons, est celui de la cité (polis), que nous ne devons pas réduire, conformément à nos représentations d'aujourd'hui, à un ensemble d'institutions politiques puisqu'elle a une fonction beaucoup plus décisive : la cité grecque n'est pas politique au sens restreint, elle constitue ce cadre culturel préalable, cet ensemble de réseaux symboliques, gestuels, culturels, langagiers, initiatiques, à partir desquels peut se constituer le suje humain comme tel. Autrement dit, c'est à la fois à la cité elle-même que le spectacle tragique est offert, et c'est à partir de ses protocoles symboliques constituants que la mise en jeu et en scène de l'énigme du mal est possible. La cité, en effet, est cela même qui se conquiert sur l'informe de l'animalité (référence constante de la parole tragique) non par des codes abstraits, mais par ou dans une subtile et interminable dialectique du même et de l'autre, du semblable et de l'étranger, du proche et du lointain, qui se trouve notamment désignée dans le mot philia. On hésite à traduire simplement ce mot par «amitié» et l'on fait bien : ce que nous appelons aujourd'hui amitié renvoie d'abord à la donnée psychologique de sentiments individuels quand il en va pour les Grecs de l'antiquité de tout autre chose, de ce pacte à la fois symbolique et affectif à partir duquel les hommes peuvent se reconnaître comme participant d'un espace commun. Symbolique : parce qu'il ne s'agit pas d'une donnée naturelle mais d'une armature de symboles susceptibles de lier ceux qui s'y trouvent constitués comme sujets. Affectif : parce que ces symboles seraient sans efficace s'ils ne se traduisaient au plan subjectif, s'ils ne s'avéraient capables de lier les affects (du désir, de l'amour et de la haine) pour produire cette forme d' «aimance» qui est à la fois la condition et la résultante du lien de la cité. La philia est en ce sens le caractère premier de toute «cité», ce qui fait d'elle le lieu de la parole, le lieu des humains. Qu'est donc la subjectivité tragique ? Ce cas d'espèce ou, mieux, ce cas extrême à 
partir duquel et grâce auquel la cité va se donner en représentation à la fois sa naissance fictive (qu'y avait-il donc «avant» la cité sinon l'informe de la déliaison ?) et son risque ou sa virtualité permanente, cette possibilité soudain de voir revenir de l'intérieur la puissance maléfique de la destruction et de la déliaison. La cité condition réelle de la tragédie, mais la tragédie mise à l'épreuve exemplaire de ce que la cité risque à chaque fois de méconnaître d'elle-même, c'est cela que l'helléniste, Jean-Pierre Vernant, par exemple, nous rappelle à bon escient. D'une part la tragédie est «un moment historique très précisément localisé dans l'espace et le temps», mais en même temps il ne faut pas comprendre qu'elle est le simple reflet de la réalité de la polis, elle est plutôt la mise à l'épreuve de sa cohérence et de ses signes, elle «fait interférer ce que la cité sépare et cette interférence est une des formes fondamentales de la transgression tragique» ${ }^{1}$.

La tragédie comme exorcisme: c'est ici que cette définition traditionnelle prend toute sa portée, en insistant sur la présence obstinée, inguérissable sans doute, de la violence sans visage et sans loi au cœur non dit de toute communauté. Le sujet tragique, en bref, est celui qui, hors cité et hors aimance, apolis et aphilos, incarne momentanément le comble du mal, sans qu'on puisse dire vraiment qu'il en va de sa responsabilité personnelle, sans qu'on puisse dire non plus qu'il n'est que le jouet de la ruine, comme si son outrance (ubris) était l'indice et la mémoire de la présence en chaque homme d'une virtualité tragique, maléfique, à contenir sans fin dans les savants protocoles de l'être-ensemble politique (politeia) et affectif (philia). Être radicalement seul, monoumenos, ne pas avoir d'ami, être dans l'outrance sauvage qui éloigne de toute cité et de tout prochain, c'est le même : en ajoutant que le propre du mal tragique, de la cruelle outrance du héros tragique, Edipe, Antigone ou Oreste, c'est d'aller jusqu'au bout de son refus de l'autre, de son refus de toute limite et de tout partage commandé par la puissance souveraine de «logos», jusqu'au bout de l'accomplissement de son vertige sauvage, sans retour et sans pardon.

Notre civilisation sans doute, notamment quand elle vient se signifier dans l'espace du théâtre, continue de regarder même obscurément vers les Grecs, vers la leçon tragique des Grecs. Il y aurait donc un invariant du mal, du rapport de l'homme au mal, qui se dirait au travers de cette forme. Pourtant, cela ne saurait aller sans tension, sans paradoxe : parce que nous sommes chrétiens en même temps (et les contemporains de Shakespeare l'étaient plus que nous), parce que nous sommes les héritiers d'une autre pensée du mal qui vient de la Bible. Or la Bible, la Bible juive, n'est pas tragique. L'humanité n'est pas ici ce qui, sous le regard des dieux de toujours, se donne dans le nouage difficile, subtil, toujours à refaire, du même et de l'autre, du proche et du lointain, de la philia et de l'aphilos : pour aller vite, nous voici dans un espace tout autre où n'y a plus ni la polis ni «les dieux». 
Ce que je me suis risqué à appeler une pensée «providentielle» du mal pour l'opposer à la pensée tragique des Grecs, n'est plus cosmique et intemporel mais s'enracine dans ce fait exorbitant que les Grecs en effet n'ont jamais pu concevoir : au commencement est la parole du Dieu unique et cette parole par delà même la Chute première fait alliance (berit), elle est ce nouage inaugural qui prélude au temps de l'histoire et qui va s'accomplir comme histoire dans la guise de la libre décision de l'homme. Il y a désormais une histoire du mal ou plutôt de la lutte, de la guerre entre le bien et le mal, entre le rapprochement de Dieu et cet éloignement de Lui qui se nomme l'idolâtrie. Une alliance qui est aussi une élection (yadôa) entre un Dieu parlant absolu qui s'adresse à l'homme (à chaque homme) par une parole faisant destin et promesse, et l'homme, à la fois comme singularité (appelée-convoquée) et comme destin générique. En bref, et je suis obligé d'être bref jusqu'au dérisoire, il y a non pas une éternité mais une tranhistoricité du mal : la puissance du mal est cela qui se déploie depuis le commencement du monde, inhérente au jeu humain, entre une histoire visible et une histoire invisible régissant la première en secret par la suréminence de l'élection. Ensuite le Très-Haut, l'Unique, est Celui qui me commande l'instance du prochain non pas comme semblable mais comme infiniment aimable selon Sa sainte volonté par delà toute image et toute idole : la verticalité de la relation commandée à l'autre en tant qu'autre s'interpose dans la latéralité toujours virtuellement meurtrière du rapport envieux-jaloux-fasciné au même, au semblable. Enfin, le sujet humain est pensé comme radicalement responsable, d'une responsabilité qui est à la foi singulière (plus de kosmos auquel adosser l'action de chacun), collective (la vérité épique du héros fait vivre au sein du nous collectif l'élection d'un peuple) et générique (puisque cette responsabilité s'inscrit dès le début dans cette grande geste méta-historique qui est donnée dans la Chute, dans l'alliance indéfiniment renouée et dans la promesse messianique, dans la promesse d'une fin des temps, d'un pardon qui serait le dernier pardon). Précisons encore qu'en regard de ce messianisme, le christianisme va introduire une inflexion majeure : la figure unique du Christ est celle qui vient s'exposer à l'extrémité du mal pour affirmer à jamais la suprématie de l'amour et du pardon sur le mal et la haine : ce mode va demeurer le lieu d'insistance d'un mal radical mais ce mal ne saurait avoir le dernier mot, il est possible en un sens de faire dores et déjà arriver en ce monde l'Autre du monde qu'est le «royaume», la vérité autre qui s'accomplira à la fin des temps dans le grand jour de la résurrection.

Et Shakespeare ? Nous devons d'abord dire de lui ce que Vernant dit des tragiques grecs ; il est dans son temps et de son temps, de ce temps de la Renaissance tardive qui est d'une certaine manière un temps «disjoint» (out of joint) en lequel se croisent une survivance très lointaine et une impasse très actuelle, qui constituent l'espace symbolique préalable à partir duquel la question de l'énigme du mal peut être soulevée. La survivance, plus complexe 
qu'il n'y paraît, est celle d'une pensée du mal qui s'enracine à la fois dans la théologie (le monarque y demeure le roi «très chrétien», «oint du Seigneur») et dans un paganisme populaire récurrent. L'impasse actuelle, c'est celle de l'individualité sceptique qui, au nom de la libre pensée, se dresse non sans panache d'ailleurs face au discours de la théologie et face aux figures «cosmiques» de l'irrationalisme populaire (les esprits, les sorcières, les magies, les sorts). Tout cela, Shakespeare ne va pas le discuter ou le réfuter, il n'est pas un philosophe, mais il va l'exposer, le faire travailler et l'expérimenter au travers des figures subjectives en dialogue de son théâtrepoème. Je dis qu'il n'est pas philosophe : il est beaucoup plus que cela peutêtre, il est celui qui met en travail la pensée du mal de son époque à travers l'exposition fictive de processus subjectifs dont aucun ne détient par lui-même la vérité, et c'est dans ce traitement singulier, sur ce mode dramatique et dialogique, qu'il nous lègue la pensée suivante : comment, au sein d'un monde toujours enraciné dans la pensée chrétienne, rendre compte d'une dimension du mal qui se trouve par ailleurs pensée dans la pensée tragique des Grecs ? En posant cette question, je n'épuise certainement pas la pensée shakespearienne du mal, puisque cette dernière se donne également dans la forme de la mise en scène historique (de ce drame des «rois» en lequel se joue le drame du devenir d'une société et d'un monde), et puisqu'elle se donne encore dans la guise de la comédie, de cette sauvagerie très réelle, menaçante, omniprésente mais reconnue et traversée à l'issue d'un rituel initiatique de la désillusion qui nous aura entraînés dans la forêt d'Ardennes ou dans celle de Windsor avec Falstaff, au royaume d'Illyrie où les ducs se meurent d'amour, au royaume d'Obéron où les reines sont amoureuses des ânes. Ma question est celle-ci : n'est-il pas vrai que le théâtre de Shakespeare nous propose à son tour un «sujet tragique» qui se caractérise, comme le sujet tragique des Grecs, par une démesure, une outrance, qui tend à le faire se retrancher de toute humanité et à parier pour une solitude et une folie sans remède, solitude du maudit par les dieux ou solitude du tyran, folie de Lear ou malfaisance de Gloucester?

Sommes-nous encore, et comment cela serait-il possible, dans le monde de l'apolis et de l'aphilos grec, ou bien y aurait-il dans le héros tragique shakespearien, Gloucester, Hamlet, Lear ou Timon, comme une tonalité très singulière liée à son mode d'affirmation de soi qui nous interdirait de le considérer sans plus comme identique à Edipe, à Antigone, à Électre ? Quelque chose qui aurait à voir à la fois avec la radicale responsabilité supposée au sujet du monde chrétien, avec l'absence de toute malédiction préalable et incompréhensible venue des dieux, enfin avec cette définition du prochain, de l'autre comme autre, qui n'est plus assurée par l'immanence civique et affective de la polis mais par la transcendance du commandement de Dieu, de ce que Lacan appelle quelque part les «commandements de la 
Parole»?

Il se trouve que je fus conduit à examiner cette question il y a quelque temps, non pas à partir du privilège accordé par Freud à Hamlet comme incarnation moderne de la névrose œdipienne ni à partir des intuitions géniales de Joyce dans Ulysses, mais à partir d'un tout petit rien presque insignifiant qui malgré tout frappait l'oreille, si l'on voulait bien revenir de la traduction française au texte original. Au fond, si les lectures de Freud ou de Joyce ne me satisfaisaient pas entièrement, si elles ne me paraissaient pas suffisantes, notamment, à susciter sur la scène le tyran Richard et sa difformité offerte à la lune londonienne, c'était en regard de cette phrase de rien prononcée par Gloucester, futur Richard III, à la fin du cycle des Henri VI : «I am myself alone» (3 Henry VI, V.6.83). Une phrase toute simple en apparence, le pronom de la première personne du singulier, le verbe être (mais nous soupçonnons, grâce à Heidegger par exemple, que le verbe être n'est pas «simple»), la forme pronominale réfléchie et cet adjectif si banal et courant en anglais, l'adjectif «alone» opposant celui qui est seul à quelque environnement communautaire. Cette phrase en apparence ne recelait aucun piège, et cependant elle résistait en moi étrangement, à la mesure de l' iinquiétante étrangeté» que je m'obstinais à y entendre, m'obstinant du même coup à supposer qu'aucun être humain dans un état de conscience normal ne pouvait être conduit à le prononcer. Une parole de défi ? Certes, et répondant à l'énormité de ce que Richard, jusqu'au moment de la défaite, se sait être en regard du commun des mortels, une «énormité» que vient signifier dans sa propre parole le mot «monstre» («deformity» est à entendre à la fois au moral et au physique, inséparablement). Richard, par là, prendrait la suite d'autres tyrans d'époques lointaines et quasi légendaires, incarnant ce que les contemporains de Shakespeare pouvaient imaginer des tyrans fameux de l'Antiquité à la mesure de leur propre férocité et de ce goût de féodaux légèrement dégénérés pour les représentations sadiques telles qu'ils en trouvaient l'occasion dans le théâtre de Sénèque. Richard serait ainsi sans mystère: rien de plus que le tyran de toujours, le sanguinaire qui défie l'ensemble du royaume et la pitié chrétienne elle-même. C'est donc en toute logique que, dès que s'annonce le revers des armes, il se retrouve seul, abandonné de tous, conformément à ce que la pensée grecque a toujours formulé à propos de la subjectivité tyrannique et de son impasse. Lecture confirmée à la fois par la scène (I.3 et VI.4) dans laquelle les trois reines offensées se réunissent pour maudire l'usurpateur, et par cette autre scène (V.3) où les victimes de sa rage meurtrière viennent le hanter à l'aube de la bataille qui va décider de son sort : en somme, la solitude («loneliness») de Richard ne serait que la sanction morale de sa tyrannie, puisqu'il est reçu des Anciens que les tyrans n'ont point d'amis. Richard apolis, telle serait la leçon.

Pourtant, cela ne va pas : parce que Richard est quelque chose de plus et 
quelque chose d'autre. Son cynisme exacerbé comporte en effet une dimension très singulière qui ne pouvait pas ne pas faire frémir le public auquel la pièce était destinée (de nos jours ce point apparaît moins) : le roi, chacun le sait, est roi de par Dieu (c'est un roi «très chrétien»), alors qu'à l'inverse le tyran accompli, et Gloucester est un tyran accompli, une figure poussée à l'extrême pour les besoins de la démonstration, est celui qui est capable de rire de Dieu lui-même, il est celui pour lequel il n'existe pas de loi autre que son désir, celui qui est «alone» au sens de «à part». Bien sûr, l'ensemble des drames historiques de Shakespeare est peuplé d'usurpateurs qui sont en un certain sens des blasphémateurs, mais nul ne blasphème aussi sciemment et systématiquement que Richard en un temps où le blasphème est puni de mort, annonçant en cela les figures extrêmes des filles de Lear. Apolis a bien ici en un sens la portée d'aphilos dans la tragédie grecque : Richard est seul en ce qu'il se présente d'emblée à nous sur la scène comme radicalement étranger, étranger à toute fraternité, à toute «brotherhood». («I have no brother, I am like no brother»). Étranger donc à une philia qui n'est toutefois plus grecque mais chrétienne : ce que Richard proclame avec un ricanement qui glace, c'est qu'il n'a pas de prochain (d'où l'insistance sur l'absence de l'amour et de la pitié : «I, that have neither pity, love nor fear»). Ni prochain comme le commande le Dieu de charité, ni semblable en tant que condition immanente de l'être-ensemble : Richard est aussi radicalement et ontologiquement seul, séparé, que les filles de Lear, sa solitude avérée est celle du «monstre». Une monstruosité subjective dont la difformité physique n'est que l'indice: craignez ma bosse ou ma claudication, je suis celui qui n'a ni égal ni semblable (ma forme est unique et hors-norme) et qui ne ressent rien de ce que vous ressentez. Cette solitude n'est donc pas exactement celle d'Antigone la sauvage, ni celle d'Oreste le matricide harcelé par les Vengeresses qui chassent la nuit, ni celle de Philoctète l'infortuné. Richard n'est nullement un monstre vomi par les dieux et l'objet de leur cruel acharnement : ce qu'il est, il ne le doit qu'à lui-même («I am determined to prove a villain»), c'est sa décision, sa volonté, son désir (le mot «will» veut dire tout cela). Entendons encore une fois ce qui parle de tranchant et de résolu dans son blasphème : «I am». Il ne s'agit pas de prendre la suite d'une très vieille malédiction ou de réclamer pour un très vieux droit ou pour un très ancien dieu face à d'autres droits et d'autres dieux : Richard n'est pas d'Edipe ni Antigone, et le drame élisabéthain n'est pas, comme la tragédie grecque, un espace de contestation dialogique, de débat argumenté entre des puissances égales. Ce que dit son «I am», si nous le couplons au «I have no», c'est : je ne dois qu'à moi d'être le monstre que je suis, je le suis parce que je veux l'être dans le mouvement même qui me retranche de tout avoir commun, c'est moi qui suis de par ma volonté «myself alone», ce solitaire inquiétant que vous voyez. Non pas seul au sens d'abandonné, mais seul parce que je décide d'être celui qui ne ressemble à 
personne («I am like no brother») et à qui personne ne ressemble. Entendezvous bien le cri que je lance ? Et si vous rétorquez que puisque je vous parle, c'est donc que je vous reconnais comme mes semblables, laissez-moi rire : je ne vous renie pas, non, je vous choque, je vous scandalise, je vous fascine, et c'est exactement ce que je veux. «Alone», je ne le suis pas exactement de vous nier, car je serais fou, mais de décider que vous n'êtes ni mes semblables ni mes égaux, et d'ailleurs je lis dans vos regards que vous m'accordez d'être précisément ce que je revendique d'être, unique. C'est là le vrai sens de ce petit «alone» que je colle à mon «myself», je suis moi-même à mes propres yeux en tant qu'unique, je me donne moi-même cet être que je revendique contre tous sans que rien puisse faire cesser cette danse vertigineuse que l'être et l'unique dansent ensemble dans mon imprécation, le «I am», le «myself» et le «alone» qui se répondent et se relancent.

$$
* * *
$$

Tout est-il dit part là, et sur la solitude, et sur Richard ? Ce «myself alone» est-il le dernier mot de la pièce ? Non, bien entendu, puisque Gloucester n'est qu'un personnage, puisque la langue de Shakespeare est ce qui vient insérer la parole de Richard dans un ensemble englobant, et ce qui répond au défi violent du tyran en l'intégrant là où il ne le sait pas et ne veut pas le savoir. Le monstre fascinant, l'unique, n'a pas le dernier mot: puisque Shakespeare n'est pas Gloucester mais celui qui le fait parler, il fait en sorte que le message que Richard avait cru pouvoir lancer sans réplique (que répliquer à «I am myself alone» ?) lui revienne comme un autre : tu es toimême tout seul, plus personne en effet pour soutenir ta cause, tu ne croyais pas si bien dire et ta vérité si bien dite (au-delà de ce que tu croyais dire) est celle de ton impasse puisque tu viens d'atteindre au point ultime de ta stérilité. Toi qui te faisais fort de ne compter que sur toi, à cette heure cruciale du jugement, de l'ordalie sous le regard de Dieu, tu ne peux compter sur rien d'autre que cet «I am» qui ne te renvoie rien, sinon le vide au miroir de toute image, - ainsi le «I am I» stupéfiant de l'acte V, scène 3, de Richard III, faitil écho dans la formulation vaine d'une vide identité, au «self against self» de la scène 4 de l'acte II. Avec ce nouage de l'unicité en impasse et de la stérilité, qui signifie l'absence d'inscription dans un ordre symbolique qui fasse loi audelà du sujet, nous touchons sans doute un possible très singulier de l'êtresujet qui se trouve libéré par la définition chrétienne du sujet comme singularité destinale libre, un possible qui secrètement éclaire toute une chaîne de personnages shakespeariens et rapproche en dépit des différences visibles Richard le tyran d'Hamlet, le prince impossible, ou de cette autre incarnation, fort énigmatique, de la solitude qu'est Timon d'Athènes.

On sait que cette pièce tardive (1606 ? 1608 ?) a toujours posé 
d'énormes problèmes aux commentateurs, lesquels ont souvent tranché au plus facile en décidant que l'œuvre n'était pas de Shakespeare ou pas entièrement de sa main, ou seulement ébauchée en vue d'une représentation qui, du vivant de l'auteur, n'eut jamais lieu. Plus sérieusement, les spécialistes et du théâtre de Shakespeare et de la culture élisabéthaine se sont efforcés de montrer de quelle manière Timon ne saurait se concevoir hors des représentations de la civilité et de l'amitié qui se trouvaient alors répandues : tout en effet conduit à poser que cette tragédie prend place dans la généalogie d'un long débat, aux époques médiévale puis renaissante, sur la question de l'amitié et de la philia entendue soit plutôt au sens de Platon (dans le Banquet), soit plutôt au sens d'Aristote (dans l'Éthique à Nicomaque) ${ }^{2}$. Il faudrait donc tenir d'emblée que ce n'est rien moins que la consistance du lien social qui se trouvait mise en cause par cette pièce. Reste la question que tout lecteur ou spectateur se pose : qui est donc Timon, d'où vient-il et comment entendre la leçon qu'il nous délivre ? Nous avons, bien sûr, le texte bref de Plutarque dans la Vie d'Antoine (69-70). Ce qui y est dit demeure fort allusif : Timon n'est nullement un héros de légende, il n'apparaît que par un biais, au détour de la mention d'une autre vie, elle légendaire. Le mode toutefois d'introduction à cette parenthèse n'est pas sans intérêt : c'est dans un moment précis de sa vie, quand il se trouve abandonné de la fortune et quitté de tous, qu'Antoine choisit de se retirer à l'écart comme Timon («Pour Antoine, il quitta Alexandrie et, renonçant à tout commerce avec ses amis, il fit construire une jetée sur la mer, non loin de Pharos, et s'y ménagea une demeure maritime où il vécut, fuyant toute société. Il aimait, disait-il, et voulait imiter la vie de Timon, dont le sort avait été semblable au sien ; car, comme lui, il avait fait l'épreuve de l'ingratitude et de l'injustice de ses amis, ce qui lui avait donné de la défiance et de la haine contre toute l'humanité»). Cette phrase d'introduction est dans le même temps phrase de conclusion: la vie de ce Timon, connu aux dires de Plutarque par Aristophane et par Platon le Comique, va servir de confirmation à ce qui vient d'être énoncé sous une forme condensée et suffisante. Antoine et Timon ont ce point en commun : ils ne sont pas d'emblée dans une position de haine et de rejet, ils n'y viennent que poussés par les circonstances, transformant subjectivement le constat d'injustice et d'ingratitude (d'aphilia de ceux qui se disaient amis) en décision résolue de «renoncer à tout commerce» et de fuir toute société. Fautil, dans l'esprit d'un Plutarque, condamner cette aphilia en y voyant un renoncement à ce qui constitue l'humanité même, faut-il le plaindre en répétant avec Aristote que rien n'est pire que d'être sans amis ? Sans doute un peu des deux, si l'on admet que la position du «misanthrope par déception» est intermédiaire entre deux autres, d'une part celle de la «retraite du sage», de la «forteresse intérieure» de l'homme stoïcien qui se retire du jeu du monde pour n'en être pas offensé, d'autre part celle du «cynique», de cet être des limites 
dont les hellénistes nous rappellent qu'il fut, tout au long de l'histoire de la cité grecque, une figure paradoxale de la rébellion visant à incarner la crudité de la «nature» en regard de la corruption de la «culture». Mais que veut dire cette dernière position dans un univers qui n'a plus rien de grec mais qui est en profondeur chrétien? Il y a bien, de ce point de vue, une énigme de la misanthropie, que désigne dans la pièce de Shakespeare la reprise du mot grec («I am Misanthropos and hate mankind») : qui n'est plus obscur, à première vue, que la possibilité d'un homme qui en viendrait à haïr l'humanité entière. D'où vient que Shakespeare s'est efforcé de dialectiser cette posture subjective, et de la répartir entre deux personnages. D'une part Timon, qui est en quelque sorte un «misanthrope devenu», non pas comme Gloucester un tyran s'affirmant d'emblée dans la négation de tout semblable mais un généreux trahi par ceux qu'il croyait amis et qui, face à cette trahison, va s'enfoncer dans un désert où les bêtes sont meilleures que les hommes («Timon will to the woods: where he shall find / The unkindest beast more kinder than mankind», IV.1). En face de Timon, Shakespeare a disposé, en se servant des indications de Plutarque, cette autre figure enracinée dans la tradition cynique, celle d'Apemantus, celui qui dès le début est présent sous la forme d'une parole dissidente au sein du triomphe mondain de Timon le généreux et qui sera de nouveau confronté à Timon du désert, à l'homme des bois misanthrope qu'il est devenu, au cours d'une joute rhétorique éblouissante qui est un des sommets de l'œuvre (scène 3 et l'acte IV). Au cours de cette scène, Apemantus met la misanthropie de Timon au compte de l'affectation, à partir de quoi le duo des deux asociaux semble tourner sur lui-même comme une toupie, un cataclysme de rage et de malédiction réciproque. Duo qui n'est pas sans rappeler, sur la lande battue des vents et giflée de pluie, celui de Lear le dément et de son fou de cour : tout comme Timon, Lear, est celui qui, par revers de fortune, est passé de la socialité à la malédiction radicale de l'asocialité, cependant que le fou qui le flanque, comme Apemantus face à Timon, relance à sa manière la parole de malédiction. Deux figures complexes : celle de Timon, parce que c'est son devenir, et son retournement, qui engendrent le drame au-delà de toute raison apparente ; celle d'Apemantus, parce qu'il est d'une part celui qui occupe depuis le commencement la fonction $\mathrm{du}$ «fool», parce que son être est avant tout de langage et qu'il est, comme le fou de Lear, comme tous les fous de Shakespeare, celui qui retourne le langage sur lui-même pour mettre à nu ses impasses secrètes, mais aussi parce qu'il ne l'est pas si simplement. Incarnant aux yeux de Shakespeare ce paradoxe vivant qu'est le cynique grec (dont il n'y a pas d'équivalent dans le reste de l'œuvre), il est constamment pris dans la contradiction du «misanthrope social», du parasite qui conteste le jeu social tout en faisant jouer sa contestation à l'intérieur du commerce communautaire (il participe au premier festin chez Timon mais sur un lit à part car «il n'aime pas la compagnie»), mais il est en 
même temps le premier à dire tout haut ce que dissimule le comportement apparent des prétendus amis de Timon ( «Je souffre de voir tant d'êtres acharnés à la curée d'un seul homme») et joue dans le même séquence le rôle du sage antique dénonçant le grand jeu des illusions mondaines («la gloire de cette vie n'est qu'une folie»)

Et Timon? Son caractère excessif, hors mesure, n'est pas sans évoquer la rage négatrice du héros tragique grec mais, ainsi que certains commentateurs l'ont relevé, il n'est pas non plus sans renvoyer à la figure biblique du juste persécuté, voire à la figure du Christ de par cette dimension sacrificielle que souligne le «tell out my blood» de l'acte III, scène 4. Figure sacrificielle et expiatoire, offerte à la dévoration haineuse de la communauté des semblables : les traits de ressemblance sont ici forts entre Timon et Lear, tous deux victimes d'un don retourné en ingratitude (et l'on pourrait encore, dans une certaine mesure, ajouter à ces deux figures celle de Bassiano qui, dans $L e$ marchand de Venise, s'offre en victime à la haine du Juif acharné à réclamer sa «piece of flesh»). Toutefois, c'est véritablement forcer les textes que de présenter Timon et Lear comme essentiellement victimes, voire même comme des figures christiques, - étrange rapprochement qui, pour les chrétiens d'alors, eût fait figure de blasphème. Étrange Christ inversé qui substitue la malédiction à la bénédiction (pas de pardon), étrange Christ surtout qui prétend recevoir en retour de ce qu'il a donné alors que le don christique est précisément le don sans compter, le don qui ne demande rien. Ce serait oublier, en somme, que si Timon et Lear sont victimes, ils sont en grande partie responsables de leur sort, comme le poète l'indique assez en leur conférant cette part de folle démesure, de démesure-folie, qui les rapproche en secret et de Richard ( «I have no brother» est une phrase de Gloucester et aussi bien de Timon ou de Lear) et du prince Hamlet («je me retire du jeu, il m'a rendu fou»). Une démesure et une folie qui tiennent à ce que la première moitié seulement de la vérité christique est entendue (celle qui dit que ce monde est celui de la haine, du mal, du désir de meurtre) au détriment de la seconde qui seule lui confère sa portée positive, non suicidaire, non gnostique (ce monde doit être racheté et «je» suis venu pour cela, pour sauver ce monde et non pour le juger). Autant dire que ces figures de la protestation et de la sécession sortent bien de l'imaginaire chrétien mais pour constituer en quelques manière la version caricaturale, fautive et même blasphématoire, de cette contestation du monde dont la révélation christique est porteuse («mon royaume n'est pas de ce monde», «qui n'est pas avec moi est contre moi», «si quelqu'un veut venir à ma suite, qu'il se renie lui-même», etc.). Dans le cas de Timon, le lieu de déploiement de sa démesure virtuelle est la sphère du don, de la fortune et de la dépense somptuaire : on tombera d'accord, en cela, avec cette remarque de Richard Marienstras que «Timon d'Athènes est une pièce qui tire parti des différences d'opinion qu'il y avait au début du 17ème siècle à 
propos des dépenses somptuaires») ${ }^{3}$. Mais justement : comment l'échange serait-il possible là où le don est si complet, si enveloppant, si extrême dans son faste que le contre-don, le don en retour, n'est plus possible? En quo l'unicité de Timon est bien parente de celle de Gloucester : que ce soit dans le registre de la parole, de la puissance politique ou de l'échange, le mal est toujours le même, c'est celui d'une dissymétrie radicale, d'une singularité telle qu'au lieu de relancer le circuit de l'échange elle le rend strictement impossible et le fait s'abîmer sur lui-même («a return exceeding all use of quittance»). Est-il au fond si aimable, le Timon du premier acte ? Que cherche-t-il sinon à donner en spectacle, comme pour apaiser on ne sait quelle secrète angoisse, sa propre excellence, la vision (voir l'importance du Peintre dans les premières scènes) de sa propre générosité qui est pour ainsi dire une générosité à fond perdu mais, à la différence du don christique, une générosité qui ne lui coûte rien et qui surtout exclut l'autre en lui interdisant toute réponse et toute riposte.

Nous ne sommes donc pas dans la perspective chrétienne qui prétend fonder en Dieu la relation au prochain (la référence à l'Antiquité a, dans Timon, la même fonction que la référence au passé légendaire dans Lear : celle d'une mise à distance de la socialité fondée dans la référence aux symboles chrétiens). Mais nous ne sommes pas non plus dans l'espace de la morale grecque: nous sommes dans l'univers de la subjectivité seigneuriale telle qu'elle survit dans les temps de la Renaissance tardive, mais délestée de tout ancrage dans ces réseaux d'obligations qu'avaient jusque là fondés la transcendance chrétienne et qu'elle était censée continuer à fonder à travers la personne du roi «lieutenant de Dieu». Timon est très clairement l'incarnation au départ d'une morale aristocratique de la dépense telle qu'elle s'oppose non pas tant à l'intérêt et à l'usure (cela, c'est ce que dit Le marchand de Venise) mais à la charge de revanche, à la réciprocité. Que Timon soit un personnage de la noblesse («nobility») et de la magnificence, le texte le fait entendre clairement: «j'ai donné imprudemment («unwisely») mais jamais ignoblement («not ignobly»)». Reste la folie de cette munificence précisément en ce point où virtuellement elle nie l'autre et reçoit dans l'évidence de l'ingratitude la monnaie de sa pièce. La leçon de Timon, en effet, est-elle simplement qu'on a raison d'être misanthrope, que les hommes décidément sont ingrats et qu'il n'est pas de véritable ami ? Si c'était cela, elle ne dirait pas grand chose, et Timon de son côté ne ferait rien de plus qu'énoncer la méchanceté du monde comme Flaminius, comme le serviteur de III.5, comme Alcibiade, dont Shakespeare fait un personnage aristocratique prenant la défense d'un autre noble, son ami. Par là d'ailleurs, Shakespeare donne déjà tort à l'excès de Timon : il y a peu de vrais amis, mais il y en a. Reste qu'il n'a pas su les voir, ce qui est déjà une faute, un signe d'aveuglement, et qu'il y a surtout cet aveuglement plus grave qui le conduit 
dans sa démesure à se poser comme l'exception unique à la malfaisance généralisée. A la verticalité généreuse et salvatrice de l'exception christique s'oppose ainsi la latéralité négative et suicidaire de l'exception misanthropique : s'il y a une énigme de la misanthropie, c'est l'énigme à la fois de l'aveuglement et de la haine. Car Timon est fautif autant que Lear et sa malédiction est aussi folle que celle de Richard dont il partage d'ailleurs un autre trait, toujours significatif chez Shakespeare, celui de la stérilité. Il est bien vrai que la pièce nous fait assister à la présence d'une sorte d'épidémie contaminant tout le corps social : c'est Athènes tout entière qui est en proie à la corruption. Mais, face à elle, Timon est un héros stérile, que Shakespeare a voulu comme un degré zéro de symbolicité : soustrait à toute généalogie, il n'a ni parents, ni femme, ni enfants, en quoi nous devons lire sa secrète faille et sa secrète négativité. Ces dernières se conçoivent mieux si nous mettons en regard de Timon d'autres subjectivités extrêmes de l'univers shakespearien. Gloucester était un scélérat qui conduisait son défi jusqu'au bout : fascination perverse, monologue de l'unique, impasse du tyran. Coriolan, de son côté, était un seigneur muré dans sa suffisance orgueilleuse et virtuellement délirante, découvrant un peu tard, mais point trop tard pour être sauvé et délivré, qu'il existe une loi supérieure à toute subjectivité et qu'il se doit comme les autres de s'y soumettre. Ces cas de figure nous éclairent, par similitude ou contraste, sur l'énigme que représente le personnage de Timon (peut-être inabouti en effet comme figure dramatique) : Gloucester était un tyran, Timon est plutôt celui qui s'en prend à la mauvaiseté généralisée et s'excepte lui-même d'un mal qu'il est impuissant à combattre, malédiction de l'impuissant plutôt que défi du pervers. Coriolan, lui, retrouve enfin d'un mouvement la fidélité à la lignée (à son nom) et à sa ville: Timon demeure stérile, récusant toute patrie puisque la patrie s'est reniée elle-même en bannissant Alcibiade, et prétendant même, malédiction récurrente du discours shakespearien, s'en prendre à la «racine de la génération».

Il y a bien dans tout cela comme une folie générale qui ne s'incarne pas dans le seul Timon : la politique elle-même est devenue impossible et la cité n'existe plus. Personne ne semble pouvoir répliquer au misanthrope et la misanthropie veut bien dire la tentation extrême de la négation de tout lien social. Reste que c'est une tentation et une folie, une folie qui culmine dans la négation du semblable comme cela ressort de l'échange verbal rapide et même tourbillonnant entre Timon l'homme des bois et Apemantus à l'acte IV. A Apemantus qui défie Timon de lui ressembler ( Do not assume my likeness»), Timon rétorque dans une sorte de cri du cœur «Were I like thee, I'd throw away myself», «si je te ressemblais, je m'arracherais de moi-même». Et Apemantus à son tour d'objecter que Timon précisément s'est détruit luimême en prétendant se ressembler («Thou hast cast away thyself, being like thyself»). Passage aussi strictement intraduisible que le «I am myself alone» 
de Richard, qui dit peut-être la même chose que cette dernière parole et peutêtre aussi autre chose : au vertige suicidaire du «thyself» en miroir s'ajoute le geste explicitement destructeur indiqué dans le «throw away» ou le «cast away», dans le mouvement dément de rejeter loin de soi celui qu'il croit ne pas être, un homme comme les autres, un semblable, au point de ne pas voir dans le meurtre de l'autre le meurtre de soi-même ${ }^{4}$. Quelle que soit sa lointaine grandeur, Timon est fou, et le déséquilibre de la pièce tient à ce que, Apemantus mis à part, il n'existe pas de personnage d'envergure pour lui donner la réplique. Il est fou, dès le début, de n'attendre rien d'autrui que la confirmation en miroir de sa propre générosité, qui n'est précisément pas une véritable générosité mais sa grimace : au désir d'être tout pour les autres, où se signe la mégalomanie latente $\mathrm{du}$ «munificent», succède, dans un retournement symétrique qui en dit la vérité, le désir tout aussi fou de n'être plus rien pour personne comme l'atteste par-delà la mort la malédiction de l'épitaphe réinventée par Shakespeare («there does not live a man») . La vérité n'est pas qu'il n'y a pas d'amis, ou qu'il y en ait peu (la pièce montre qu'il y en a quelques uns, ce qui suffit), elle est que l'amitié suppose la charge de revanche et la parole qui engage des deux côtés. Que fait Timon pour les autres sinon les couvrir de présents ? Et comment est-il assez fou pour imaginer que des obligés sont par là même des amis ? De quel droit enfin juge-t-il ceux qu'il estime être en dette vis-à-vis de lui et quelle est donc cette rente qu'il croyait avoir sur la générosité des autres ? Richard Marienstras a encore parfaitement raison quand il note finement que «Timon semble n'offrir ses dons que pour se prouver à lui-même que cette société régie par l'amitié existe réellement». C'est là sa faille, en conclusion, c'est là sa faute : de passer sans transition de l'extrême de l'illusion à l'extrême de la négation du monde, ces deux extrêmes se réciproquant en une prétention délirante. En quoi nous comprenons bien que cette figure insituable ait plu à Shakespeare et qu'il l'ait abordée dans le même mouvement que le vieux roi Lear, situé lui aussi hors d'un temps délimité, dans un espace de légende : il fallait que, tout comme Lear, Timon crût follement que ce monde pouvait reposer sur la transparence d'une réciprocité généreuse, il fallait aussi, pour alimenter sa folie, que la méchanceté fût effective et l'ingratitude quasi générale, autrement dit qu'il pût tout comme Lear dénoncer une vérité mais se perdre du même coup en la prenant pour la vérité «toute», et s'imaginer être celui qui pouvai la dire toute sans y être compté. Démesure grecque ? Assurément non : il n'y aura ici ni persécution par aucun dieu, ni ligature de l'être individué par une mauvaiseté clanique qui le suit pas à pas et dont il hérite malgré lui. Timon est un sujet moderne en ce qu'il ne trouve qu'à l'intérieur de lui-même la ressource et de son aveuglement et de sa malédiction, moderne encore en ce qu'aucun Dieu ne surplombe sa parole et son action, moderne enfin en ce qu'il accomplit une possibilité subjective que les Grecs ignoraient et que le discours 
chrétien rend possible au contraire, cette négation du monde libérée par la révélation (hétérogène à tout «cosmisme») que ce monde dans sa totalité peut être contesté et suspendu au nom d'une parole autre, d'une vérité autre qui n'est pas du monde. Je veux dire que ce Timon élisabéthain, forgé à partir d'une antiquité de légende, d'un archaïsme insituable, n'a entendu, comme je le suggérais en commençant, que la moitié de la révélation christique, messianique, et qu'il est en cela hérétique : il entend que ce monde est marqué radicalement par le péché, que la haine sans cesse y fait son œuvre et trouve des victimes, que l'innocence est bafouée, que le juste est persécuté... mais il oublie la fin de la phrase, qu'il suffit d'un juste pour témoigner que cette méchanceté ne se referme pas sur elle-même, que le monde, ce qu'on appelle le monde (le spectacle du monde), est divisé, que le Messie enfin est venu pour affirmer la lumière et la véracité du «royaume» au sein du «siècle».

D'une manière frappante Timon incarne en somme, dans la succession de ses deux postures (que Shakespeare imagine car Plutarque n'en dit rien) l'alternance symétrique, aux premiers temps de la parole chrétienne, des doctrines apocalyptiques et des doctrines gnostiques, l'illusion d'une part que ce monde-ci peut dores et déjà se trouver régi par l'autre vérité en devenant «le royaume», la conviction inverse que ce monde intrinsèquement mauvais doit être abandonné à sa malfaisance sans remède, ontologique, et qu'il convient de rejoindre ailleurs la région du salut. Timon est celui qui a cru à la transparence accomplie du bien et qui, par dépit, comme un enfant se met en colère, régi par la loi primaire du tout ou rien, rejette ce qu'il avait d'abord adoré mais sans croire en aucun salut (d'où le malaise que suscitent et Timon et King Lear en se renfermant sur le vide). Un gnostique qui ne croirait en aucun audelà, telle est la position de Timon. Pièce noire absolument, qui semble renvoyer dos à dos sans aucune position tierce (car, tout de même, dans Lear il y a Kent, et le fou et Edgar, et la destruction interne des méchants) le misanthrope professionnel, cet Apemantus, parasite logé au sein du corps social comme le ver au cœur du fruit, et ce Timon dont l'être-sujet s'inverse d'un excès de crédulité intéressée, narcissique, en une désillusion suicidaire. Sorte de cas de figure chimiquement pur servant à démontrer la structure en impasse de toute malédiction qui ne saurait se porter contre l'ensemble des autres, du monde, sans être aussi malédiction contre soi. De tous les personnages de Shakespeare, Timon est, avec Lear, celui qui va le plus loin dans la négation puisque ne le motivent ni la soif de pouvoir, comme Gloucester, ni la fidélité au père, comme Hamlet, ni l'amour de la gloire, comme Coriolan. Mais ne désire-t-il pas la reconnaissance, ne désire-t-il pas être aimé ? Voilà en effet sa folie propre : car il ne peut pas ne pas nous apparaître comme celui qui ne sait pas aimer. Lui qui passe son temps à donner ce qu'il a, ce qui ne vaut guère à entendre le Christ des Évangiles, n'a pas compris que, pour se faire aimable, il faut apprendre surtout à donner ce 
qu'on n'a pas.

Bernard S ICHÈ RE

Université Paris VII - Denis Diderot

\section{${ }^{\mathbf{N}}$ O T E S}

${ }^{1}$ Jean-Pierre Vernant, «Edipe sans complexe», in J.-P. Vernant et J.-P. Vidal-Naquet, Mythe et tragédie en Grèce ancienne, I, Maspero, 1972.

${ }^{2} \mathrm{Je}$ me réfère ici à un article à paraître de Richard Marienstras, «Mesure et démesure dans Timon d'Athènes».

${ }^{3}$ Ibid.

${ }^{4}$ Cette logique suicidaire du narcissisme a été magnifiquement exposée par Lacan, à propos du personnage de l'Alceste de Molière, dans «Propos sur la causalité psychique», in Écrits, Seuil, 1966. 